\title{
Sugary Endosperm is Modulated by Starch Branching Enzyme lla in Rice (Oryza sativa L.)
}

\author{
Yunjoo Lee ${ }^{1}$, Min-Seon Choi ${ }^{2}$, Gileung Lee ${ }^{1}$, Su Jang ${ }^{1}$, Mi-Ra Yoon ${ }^{3}$, Backki Kim', Rihua Piao ${ }^{4}$, Mi-Ok Woo',
} Joong Hyoun $\mathrm{Chin}^{5^{*}}$ and Hee-Jong Koh ${ }^{1^{*}}$ (D)

\begin{abstract}
Background: Starch biosynthesis is one of the most important pathways that determine both grain quality and yield in rice (Oryza sativa L.). Sugary endosperm, sugary-1 (sug-1), is a mutant trait for starch biosynthesis. Rice plants carrying sug- 1 produce grains that accumulate water-soluble carbohydrates instead of starch, even after maturity. Although this trait enhances the diversity of grain quality, sugary endosperm rice has hardly been commercialized due to the severely wrinkled grains and subsequent problems in milling. This study was conducted to identify the genes responsible for the sug-h phenotype through a map-based cloning technology.

Results: We induced a mild sugary mutant, sugary-h (sug-h) through the chemical mutagenesis on the Korean japonica cultivar Hwacheong. Grains of the sug-h mutant were translucent and amber-colored, and the endosperm appeared less wrinkled than sug-1, whereas the soluble sugar content was fairly high. These characteristics confer greater marketability to the sug-h mutant. Genetic analyses indicated that the sug- $h$ mutant phenotype was controlled by a complementary interaction of two recessive genes, Isoamylasel (Os/SA1), which was reported previously, and Starch branching enzyme Ila (OsBElla), which was newly identified in this study. Complementation tests indicated that OsBElla regulated the properties of sugary endosperm.

Conclusions: Complementary interactions between the starch biosynthesis genes OsISA1 and OsBElla determine the mild sugary endosperm mutant, sugary-h, in rice. Our finding may facilitate the breeding of sugaryendosperm rice for commercial benefit.
\end{abstract}

Keywords: Map-based cloning, OsBElla, OsISA1, Rice, Sugary-h, Sugary endosperm

\section{Background}

Rice is the staple food for more than 3 billion people globally. The endosperm is an edible part of the rice grain, and has been one of the major targets for grain geneticists and breeders to enhance grain yield and quality. Endosperm development directly regulates grain formation at the grain filling stage. Mature rice endosperm contains starch, storage proteins, lipids, and other substances. Studies on starch have been an essential

\footnotetext{
* Correspondence: jhchin@sejong.ac.kr; heejkoh@snu.ac.kr

${ }^{5}$ Graduate School of Integrated Bioindustry, Sejong University, 209,

Neungdong-ro Gwangjin-gu, Seoul, South Korea

${ }^{1}$ Department of Plant Science and Research Institute for Agriculture and Life

Sciences, and Plant Genomics and Breeding Institute, Seoul National

University, Seoul 08826, South Korea

Full list of author information is available at the end of the article
}

focus in rice research. Starch is the primary component that makes cereal crops economically and commercially important. Starch research is also becoming increasingly relevant for industrial and manufacturing applications.

Rice starch is composed of amylose (linear $\alpha-1,4$ polyglucans) and amylopectin ( $\alpha$-1,6-branched polyglucans). Amylopectin has a distinct fine structure called multiple cluster structure, and accounts for approximately $65-85 \%$ of storage starch (Nakamura 2002). Starch is synthesized by four enzyme classes, with multiple subunits in each class: ADP-glucose pyrophosphorylase (AGPase); starch synthase (SS); starch branching enzyme (BE); and starch debranching enzyme (DBE). Other enzymes, such as phosphorylase (Pho) and disproportionating enzyme, are thought to be involved in starch biosynthesis. $\mathrm{BE}$ and 
DBE have important roles in determining amylopectin structure. BE forms the $\alpha-1,6$-glycosidic bonds of amylopectin, whereas DBE trims improper branches generated by BE (Fujita 2014).

$\mathrm{BE}$ isoforms are classified into two groups, BEI (RBE1) and BEII. Cereals have two BEII isozymes, BEIIa (RBE4) and BEIIb (RBE3). These isoforms are classified according to the transferred amylopectin chain length. For example, BEII transfers shorter chains than BEI, and BEIIb transfers shorter chains than BEIIa, during extended incubations (Mizuno et al. 2001). The expression patterns of $\mathrm{BE}$ isoforms also differ. BEI and BEIIa transcripts have been localized in the endosperm and other tissues, whereas BEIIb is expressed only in the endosperm and reproductive tissues. In rice, BEIIa is expressed earlier than either BEIIb or BEI (Mizuno et al. 2001; Ohdan et al. 2005). Previous reports designated BEIIb-deficient mutants in maize and rice as amylose-extender (ae) mutants, in which the abundance of short amylopectin chains was reduced (Kim et al. 1998; Nishi et al. 2001). Other transgenic research about BEIIb gene was reported that the manipulation of BEIIb activity can generate various starch type rice, containing chalky and sugary endosperm (Tanaka et al. 2004). The RNA interference results demonstrated that reduced expression of BEIIa (SBEIIa) caused increase of amylose content in wheat endosperm (Regina et al. 2006). However, the specific functional role of BEIIa has not been elucidated in rice because the seed phenotypes of BEIIa-deficient mutants and wild-type plants are not significantly different (Fujita 2014).

DBEs directly hydrolyze $\alpha-1,6$-glycosidic linkages of $\alpha$-polyglucans. DBEs are classified into two types in higher plants, Isoamylase (ISA1, ISA2, and ISA3) and Pullulanase (PUL). According to Fujita (2014), Isoamylase1 (ISA1)-deficient mutants (isa1) were called as sugary-1 mutants in rice (sug-1) and maize (su1). These mutants have a defect in the amylopectin cluster structure, which results in the accumulation of a polymeric water-soluble polysaccharide (WSP) termed phytoglycogen, and a reduction in the starch content (James et al. 2003). There are various sug-1 mutant types, EM series, reported by Japanese group (Nakamura et al. 1997; Nakamura et al. 1996; Wong et al. 2003). The sug-1 locus in rice is located on chromosome 8 (Fujita et al. 1999; Yano et al. 1984). In transgenic sug-1 rice expressing the wheat ISA1 gene, phytoglycogen synthesis is substantially replaced by starch biosynthesis in the endosperm (Kubo et al. 2005). This result implies that ISA1 is essential for amylopectin crystallinity and biosynthesis in both rice and wheat. In maize, double mutant defective in both ISA2 and SSIII generated watersoluble glucans in the mutant endosperm, although single mutants of either ISA2 or SSIII could synthesize normal amylopectin (Lin et al. 2012). By contrast, the contribution of PUL for amylopectin trimming was much smaller than that of ISA1, and PUL function partially overlaps with that of ISA1 (Fujita et al. 2009).

Since rice grains of $\operatorname{sug}-1$ mutants primarily contain water-soluble carbohydrates instead of starch even after maturity, they have the potential value in breeding programs for diversified qualities and commercial uses. However, they have not been used in practice due to the severely wrinkled grains and subsequent problems in milling. We developed a mild-type sugary mutant in rice, sugary-h (sug-h), which displayed an intermediate phenotype between the sug-1 mutant and wild type. Grains of the sug- $h$ mutant have better quality for subsequent processing and higher yield than sug-1. In addition, palatability, protein and amylose content which are crucial for breeding were increased in sug- $h$ mutant (Yoon et al. 2009; sug2 was renamed as sug- $h$ to avoid confusion with Nakagami et al. (2016)). Therefore, sug- $h$ mutants could be valuable for practical applications and nutritional aspects. This study performed map-based cloning to identify the genes responsible for the sug- $h$ phenotype. Our results not only identified a potential resource for utilizing the sugary endosperm mutant for commercial benefit, but also may provide a new insight into starch biosynthesis.

\section{Results}

\section{Phenotypic Characterization of the sug-h Mutant}

The sug-h mutant did not exhibit abnormal phenotypes during the vegetative stage of plant growth, although the heading date was delayed and plant height was reduced compared with that of wild-type plants (Table 1; Additional file 1: Figure S1). Grains of the sug-h mutant displayed an intermediate phenotype between the wildtype and sug-1 mutant grains, that was medium thickness and slightly wrinkled (Fig. 1a-f). Wild type, sug-1, and sug- $h$ mutants showed significant differences in grain dimensions and thickness. The grain length and width of sug- $h$ mutant was slightly longer and shorter, respectively, than that of wild-type (Table 1).

\section{Morphological Properties of Starch in Mutant Endosperm} Grains were stained with iodine to identify starch components. Phytoglycogen, which does not stain with iodine, had the highest abundance in sug-1 endosperm. The sug- $h$ mutant grain was partially stained in outermost endosperm layers, whereas the entire wild-type endosperm was stained (Fig. 1g-i). These results indicate that the endosperm starch components in both sugary mutants differed from those in the wild type, and starch production was at least partially restored in the sug-h endosperm.

Cross sections of polished rice grains were observed with scanning electron microscope (SEM). The images revealed that sug-1 and sug-h mutants had loosely packed, abnormal starch granules in the cutting plane compared with the densely packed granules in wild-type 
Table 1 Agronomical characters of plant and dimensions of grain in wild type (Hwacheong) and mutants

\begin{tabular}{|c|c|c|c|c|c|c|c|c|c|}
\hline Trait & $\mathrm{HD}$ & $\mathrm{CL}(\mathrm{cm})$ & PN & SN & TGW (g) & $\mathrm{GL}(\mathrm{mm})$ & $\mathrm{GW}(\mathrm{mm})$ & GT (mm) & GS \\
\hline WT & Aug.17 & $85.90 \pm 1.61^{a}$ & $14.60 \pm 1.43^{b}$ & $98.90 \pm 16.97$ & $19.88 \pm 0.19^{a}$ & $5.11 \pm 0.09^{b}$ & $3.06 \pm 0.07^{b}$ & $2.17 \pm 0.03^{c}$ & $1.67 \pm 0.54^{a}$ \\
\hline sug-1 & Aug.20 & $87.90 \pm 3.04^{b}$ & $11.10 \pm 3.57^{\mathrm{a}}$ & $90.30 \pm 10.86$ & $8.76 \pm 0.30^{b}$ & $4.89 \pm 0.09^{a}$ & $2.96 \pm 0.09^{a}$ & $1.07 \pm 0.13^{\mathrm{a}}$ & $1.65 \pm 0.05^{a}$ \\
\hline sug-h & Aug.20 & $75.60 \pm 1.31^{c}$ & $16.40 \pm 1.84^{b}$ & $101.80 \pm 9.78$ & $14.52 \pm 0.15^{c}$ & $5.41 \pm 0.13^{c}$ & $2.91 \pm 0.10^{a}$ & $1.70 \pm 0.11^{b}$ & $1.86 \pm 0.07^{b}$ \\
\hline
\end{tabular}

$H D$ heading date, $C L$ culm length, $P N$ number of panicles per plant, $S N$ number of spikelets per panicle, TGW 1000-grain weight for brown rice, $G L$ grain length, GW grain width, GT grain thickness, GS grain shape (length/width), WT wild type (Hwacheong), sug-1 sugary-1, sug-h sugary-h

Different letters denote significant differences. Ten biological replicates were used to measure for each of the traits

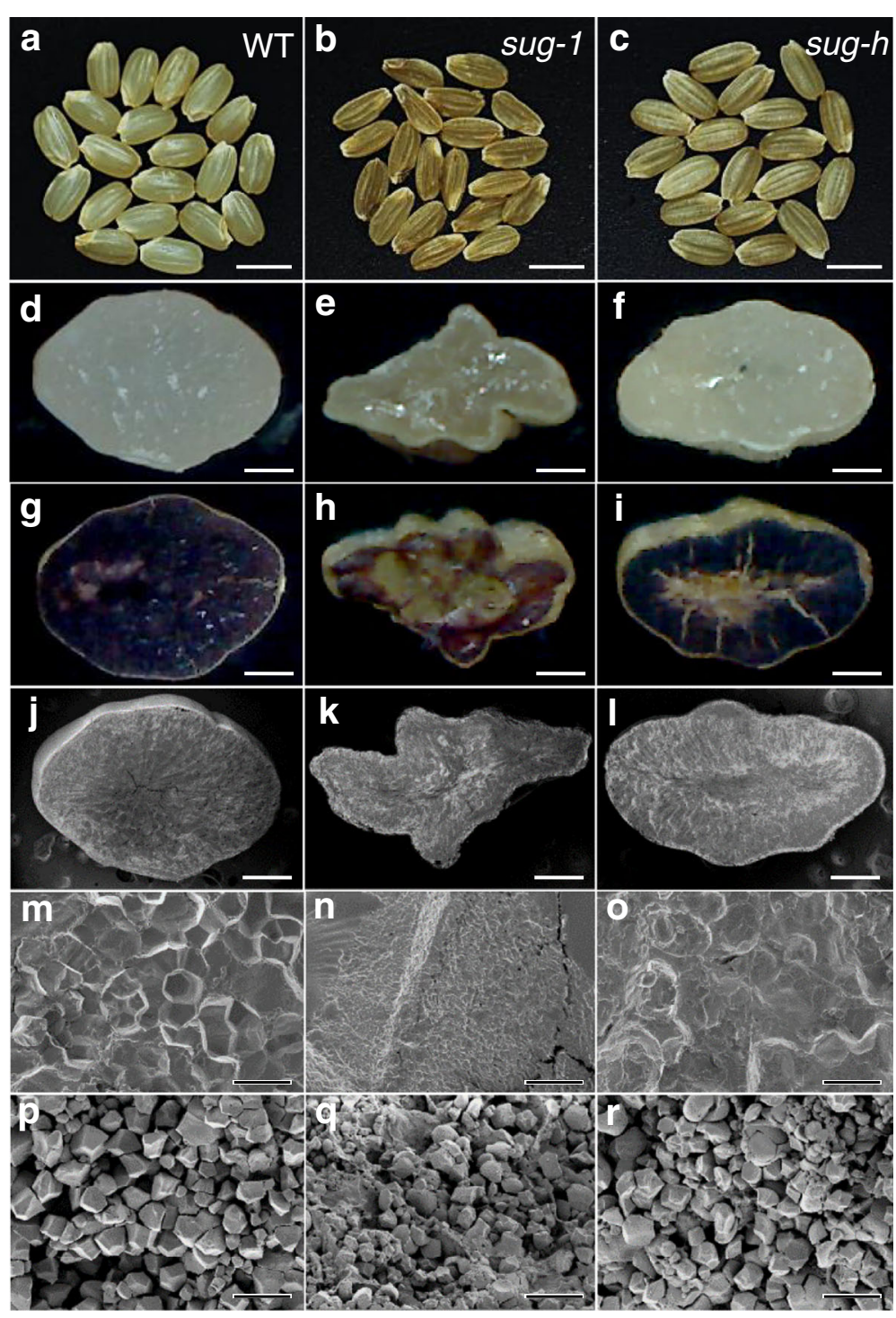

Fig. 1 Optical and scanning electron microscopy observation of grain phenotype and starch granule structure. a-c The sug-h mutant grain exhibits a phenotype that is intermediate between that of the wild type and sug-1. Bars $=4 \mathrm{~mm}$. $\mathbf{d}-\mathbf{i}$ Cross sections of wild-type, sug-1, and sug-h kernels at the mature stage. Sectioned seeds were stained with iodine solution. Bars $=0.5 \mathrm{~mm}$. $\mathbf{j}$-o SEM observations of wild-type, sug-1, and sug-h mutant endosperm. j-l Bars $=0.5 \mathrm{~mm}$. (m-o) Bars $=10 \mu \mathrm{m}$. p-r SEM observations of starch granule structures in wild-type, sug- 1 , and sug-h mutant endosperm. Bars $=10 \mu \mathrm{m}$ 
polished grains. The sug- $h$ endosperm had starch granule packing that was intermediate between that of sug-1 and the wild type (Fig. 1j-o). Starch granules in sug-1 and sug$h$ mutants also displayed irregular shapes with round edges, whereas those in the wild type displayed polygonal shapes with sharp edges (Fig. 1p-r). These results indicate that aberrant starch production was involved in the abnormal phenotypes of sug- 1 and sug-h mutants.

Starch crystallinity was investigated by performing $\mathrm{X}$-ray diffraction analysis of endosperm starch from the wild type, sug-1, sug-h, and Sug-1/sug-h. Diffraction patterns of sug- 1 and sug-h starch displayed lower peak intensity than that of the wild type (Fig. 2). These X-ray diffraction patterns indicate that the crystallization of sug- 1 and sug-h starch was lower than that of wild-type starch, as reported by Yoon et al. (2009). Starch from sug-h mutant had an intermediate crystallization level between that from sug-1 and the wild type. However, Sug-1/ sug- $h$, which has a normal phenotype, displayed similar starch crystallinity to that of wild-type starch. These results indicate that sug- 1 is a major effect on the starch structure and crystallization.

\section{Amylopectin Fine Structure in Mature Mutant Endosperm} Fine structural feature of amylopectin in wild-type and mutant endosperms were compared in detail by analysis of amylopectin chain-length distribution using high performance anion-exchange chromatography and a pulsed

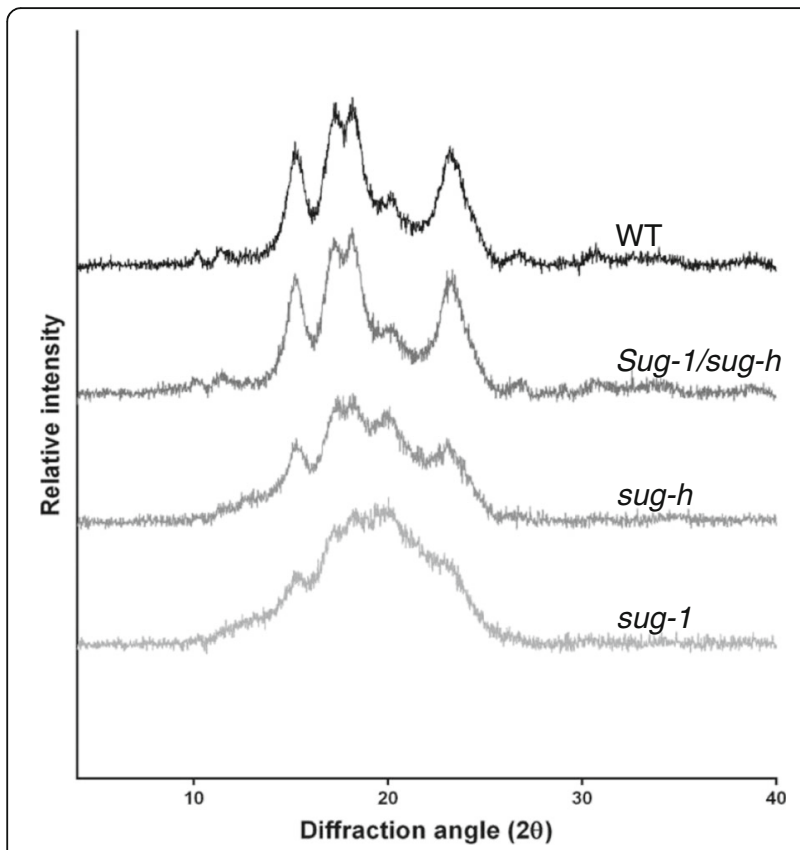

Fig. 2 X-ray diffraction analysis of purified starch granules from mature endosperm of wild-type rice (Hwacheong), sug-1, sug-h, and Sug-1/sug-h. The seeds possessed a normal OsISA1 and a mutated osbe2a genotypes were designated as Sug-1/sug-h pulsed amperometric detector (HPAEC-PAD). Both sugary mutants had the definitely increased amount of short chains in the range of DP 6-10 (Fig. 3a-d). This result was similar to the previous reports (Nakamura et al. 1997; Wong et al. 2003; Yoon et al. 2009), indicating that mutation on ISA altered the fine structure of amylopectin into phytoglycogen in rice endosperm. The difference between sug- 1 and sug-h on fine structure of amylopectin was relative amount of short chains. Most range of chain-length distribution of amylopectin in sug- $h$ was similar with that of sug-1, except in the range of DP 7-8 and DP 13-17 (Fig. 3e-f). Structural feature of amylopectin suggest that the sug- $h$ phenotype was determined by the relative amount of short chains.

\section{Map-Based Cloning of Genes Related to the sug-h Phenotype}

We performed map-based cloning to elucidate the genes related to the $s u g$ - $h$ phenotype. Normal-type seeds were designated as 'N-type' and sugary-type seeds were designated as 'S-type'. S-type seeds were grouped into 'I-type' (sugary-1 type), mixed-type (I-type and II-type), and 'II-type' (sugary-h type) in segregated populations. $F_{2}$ seeds derived from a cross between the sug-h mutant and Hwacheong were used for segregation ratio analysis. The results from $352 \mathrm{~F}_{2}$ seeds identified $271 \mathrm{~N}$-type, 57 I-type, and 24 II-type seeds, which fit the expected ratio of 12:3:1 $(P=0.45)$ (Additional file 2: Table S1). Expected genotypes of N-type, I-type and II-type will be Sug-1_Sug-h-, sug-1sug-1Sug- $h_{-}$, and sug-1sug-1sug-h sug-h, respectively. Based on this result, we hypothesized that the sug- $h$ phenotype was controlled by the epistatic interaction between two genes. One of these genes is the preceding gene, which is related to the sugary endosperm phenotype, and the other is the interacting gene, which reduces the severity of the sugary abnormality in starch biosynthesis.

Preliminary mapping was initially conducted on $352 \mathrm{~F}_{2}$ plants derived from sug-h/Milyang.23 (M.23, a Korean tongil-type cultivar), and mapped the first gene related to the sugary phenotype. Bulked DNA for the first bulked segregant analysis (BSA) was prepared to distinguish $\mathrm{N}$-type, heterozygous type (N-type and S-type), and Stype plants in the $F_{2}$ population. Two of seventy-two sequence tagged site (STS) markers across all chromosomes, S08105 and S08107 on chromosome 8, were used as the flanking markers for fine mapping (Fig. 4a). Eighteen genes were identified in the fine-mapped region. The OsISA1 gene sequence (Os08g40930) of Hwacheong and sug-h mutant was primarily compared, because previous studies reported that OsISA1 affected the sugary phenotype in sug1 rice (Kubo et al. 1999; Nakamura et al. 1992; Nakamura et al. 1996). Our sequence analysis revealed that nucleotide residue 6179 in OsISA1, which was adenine (A) in the wild type, was substituted with guanine (G) in sug-1 and sug- $h$ 

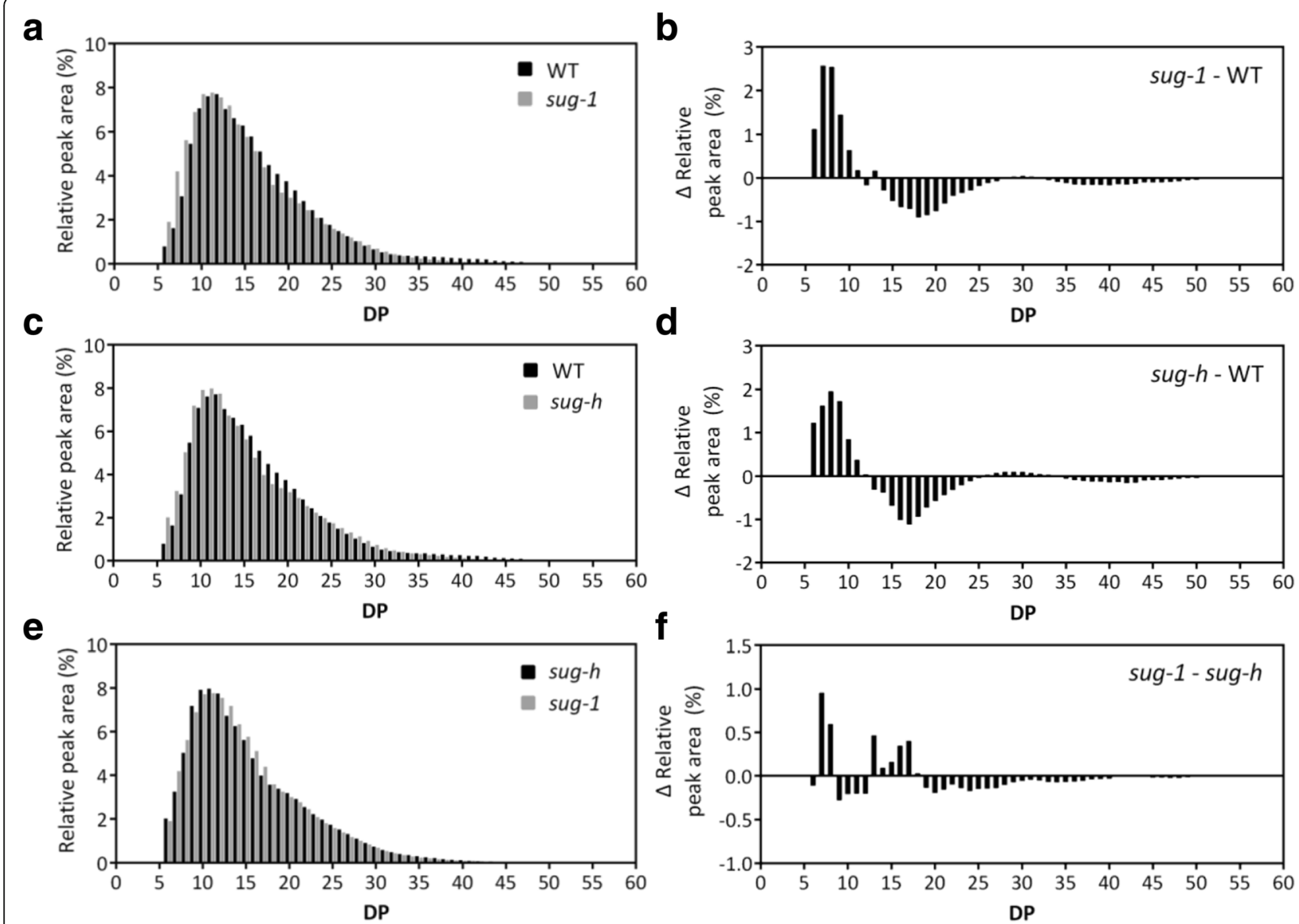

Fig. 3 Comparison of chain length distribution of amylopectin in rice endosperm between wild type (Hwacheong) and mutant. The left panels showed chain-length profiles, and the right panels showed differences in chain-length profiles. Chain-length profiles after debranching the starch fraction and differences between WT and sug-1 mutant (a-b), between WT and sug-h mutant (c-d), and between sug-1 and sug-h mutant (e-f) were shown

mutants, thereby changing the amino acid from glycine (Gly) to aspartic acid (Asp). These results indicate that the sugary endosperm phenotype could be caused by a point mutation on OsISA1, which was shared by both sug-1 and sug-h mutants.

One of the objectives in this study was to identify the second gene controlling the thickness and wrinkling of the sugary endosperm. Therefore, $837 \mathrm{~F}_{3}$ plants were derived from two individual $\mathrm{F}_{2}$ plants in which the osisa1 allele was fixed, for efficient mapping. Bulked DNA for the second BSA was prepared from I-type, mixed-type (I-type and II-type), and II-type plants selected from the $\mathrm{F}_{3}$ population. The second BSA revealed that markers and the sug-h phenotype co-segregated on chromosome 4 , indicating that the second gene was located on chromosome 4. For fine mapping, S04056A and S04058 were selected as flanking markers. One STS marker, S04057, and two dCAPS markers, AL731A and AL731B within the AL731641 clone, were additionally designed and applied for linkage analysis. These results reduced the size of the candidate region to $12.9 \mathrm{~kb}$, which contained the following three genes: 1,4- $\alpha$-glucan-branching enzyme 2; Ser/Thr protein phosphatase family protein; and histone deacetylase (Fig. 4b). These genes were sequenced and compared in Hwacheong and sug- $h$ mutant, and a point mutation was detected in the 1,4- $\alpha$ glucan-branching enzyme 2 (OsBEIIa; OsSBE4) gene of the sug-h mutant. We found that the nucleotide residue 4732 in wild-type OsBEIIa, which was G, was substituted with $\mathrm{A}$ in the sug-h mutant, thereby changing the amino acid from Gly to Asp. This amino acid substitution was not found in other grain species including maize, sorghum, barley, and wheat (Additional file 3: Figure S2). These combined results indicate that the sug-h mutant might be caused by single point mutations resulting in amino acid substitutions in both OsISA1 and OsBEIIa.

\section{Transgenic Complementation of the sug- $h$ Mutation}

To confirm the function of OsBEIIa in the sug- $h$ mutant, we generated dsRNA-mediated interference (RNAi) 


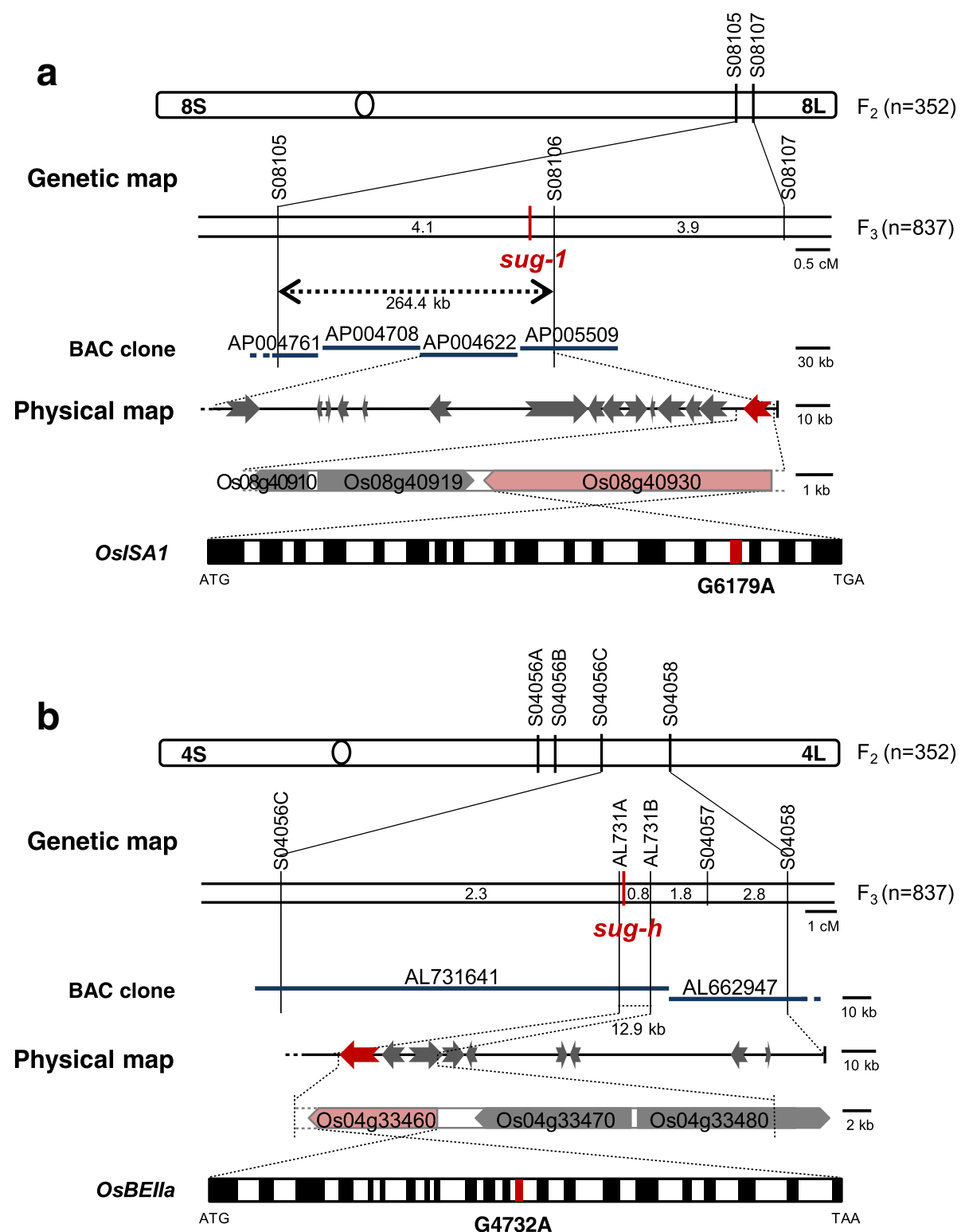

Fig. 4 Map-based cloning of the sug-h mutant. a Candidate region of the sugary gene was on the long arm of chromosome 8. Candidate gene of the first bulked segregant analysis, Os08g40930, was located on the AP005509 BAC clone and contained 18 exons (black boxes) and 17 introns (white boxes). A point mutation, $\mathrm{G}$ to A, occurred in the 15th exon (red box). b Schematic representation of OsBElla on the long arm of chromosome 4. Candidate region of the gene related to the sug-h mutant was located within the AL731641 clone. The physical distance was approximately $12.9 \mathrm{~kb}$, which included three candidate genes. Schematic structure of the candidate gene, Os04g33460, contained 22 exons (black boxes) and 21 introns (white boxes). A point mutation, G to A, occurred in the 13th exon (red box). Gray arrows indicate the main genes with known functions within the candidate region. Red arrow indicates the candidate gene. ATG and TGATAA indicate the initiation and termination codons, respectively

transgenic plants. $\mathrm{T}_{1}$ seeds of the OsBEIIa-RNAi transgenic line had normal phenotype, suggesting that a single mutation of OsBEIIa had no effect on seed phenotype (Fig. 5a). To evaluate interactions between OsISA1 and OsBEIIa, artificial crossing was performed between the OsBEIIa-RNAi $\mathrm{T}_{0}$ plant and the sug-h mutant. After phenotypic selection, $F_{2}$ seeds from the artificial cross were planted for co-segregation analysis of phenotype, genotype, and RNA expression. Phenotypes of segregated $\mathrm{F}_{2}$ seeds showed that $\mathrm{N}$-type seeds were slightly thinner than wild-type (Dongjin) seeds. By contrast, the thickness of I-type and II-type seeds was not significantly different from that of sug-1 and sug-h seeds, respectively (Fig. 5b). Genotype analysis using an antibiotic resistance gene-specific primer showed that PCR bands were produced in all II-type plants, but no bands were amplified in all I-type plants (data not shown). The qRT-PCR analysis indicated that the relative OsBEIIa 

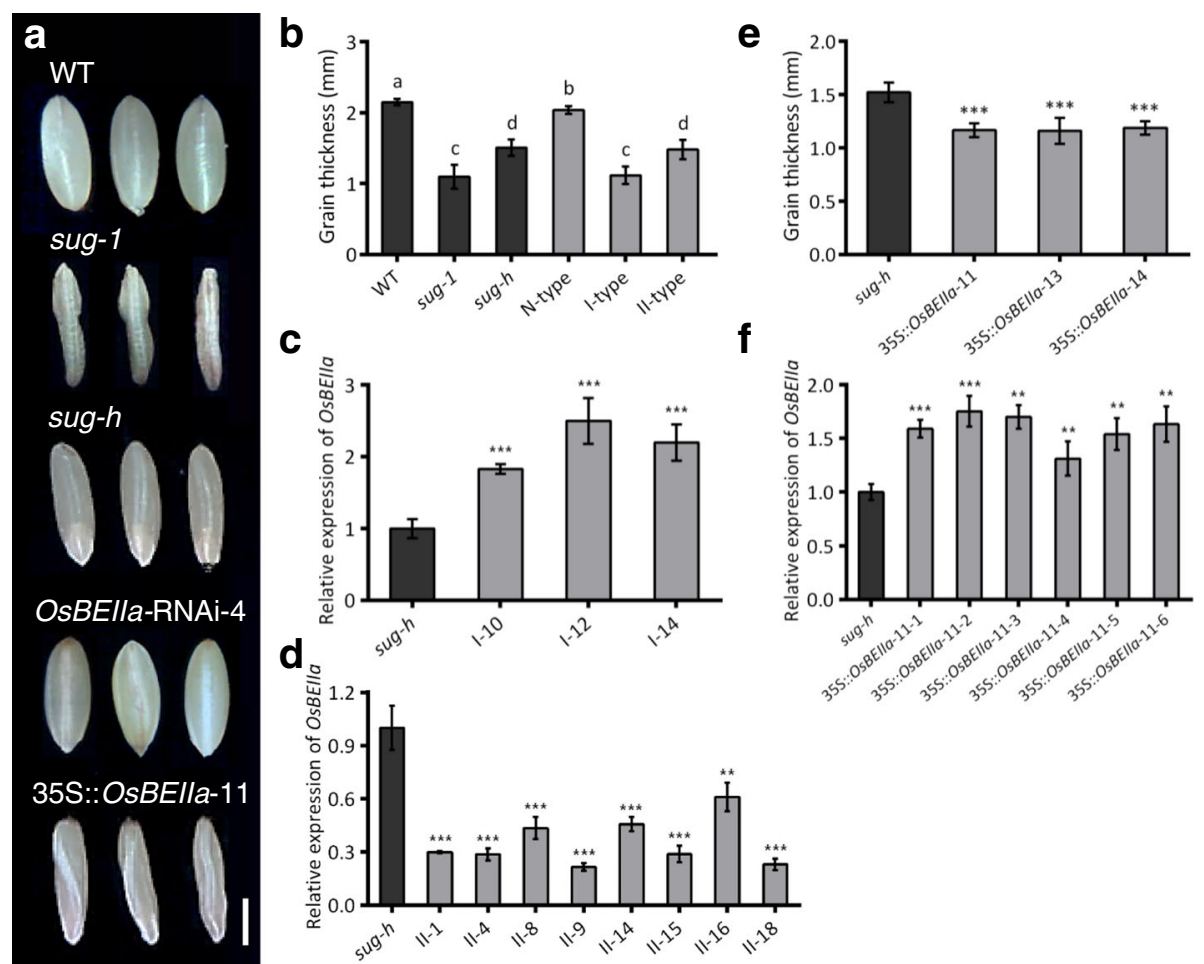

Fig. 5 Phenotype of transgenic seeds and complementation test of the sug-h mutation. a Grain morphologies of wild-type rice (Dongjin), sug-1, sug-h, and transgenic seeds. Bar $=2 \mathrm{~mm}$. $\mathbf{b}$ Comparison of grain thickness in $F_{2}$ seeds derived from OsBElla-RNAi-4/sug-h. Different letters denote significant differences. Replicate samples were measured 20 times. c Relative expression level of OsBElla in each $F_{2}$ plant derived from I-type seeds using qRT-PCR. The sug-h mutant was used as a control. Error bars represent SD for three technical experiments. Asterisks indicate statistical significance compared with the control, as determined by Student's $t$-test (***P $<0.001$ ). $\mathbf{d}$ Relative expression level of OsBElla in each $F_{2}$ plant derived from II-type seeds by qRT-PCR. Error bars represent SD for three technical experiments. Asterisks indicate statistical significance compared with the control, as determined by Student's $t$-test $\left.{ }^{* *} P<0.01,{ }^{* * *} P<0.001\right)$. e Comparison of grain thickness in three lines of 35S::OsBElla and sug-h mutant seeds. Statistical significance was determined by Student's $t$-test ( $\left.{ }^{* * *} P<0.001\right)$. Replicate samples were measured 20 times. $\mathbf{f}$ qRT-PCR analysis showed increased OsBElla expression in 35S::OsBElla-11 $\mathrm{T}_{1}$ plants. Error bars represent SD for three technical experiments. Statistical significance was determined using Student's t-test $(* * P<0.001)$

expression levels were higher in each $F_{2}$ plant derived from I-type seed than in the sug-h mutant (Fig. 5c), whereas the relative OsBEIIa expression levels in each $\mathrm{F}_{2}$ plant derived from II-type seed were lower than that in the sug-h mutant (Fig. 5d). These results show that phenotype, genotype, and RNA expression co-segregate in the OsBEIIa-RNAi-4/sug- $h \mathrm{~F}_{2}$ population, indicating that the two genes are associated with the sug- $h$ phenotype.

We also generated transgenic plants overexpressing OsBEIIa, in which a vector was introduced into the sug- $h$ mutant to complement the phenotype. According to the mapping results that the sug- $h$ mutant might be caused by single point mutations in both OsISA1 and OsBEIIa genes, this complementation test was intended to reconstruct the sug- 1 phenotype from sug- $h$ mutant. Most $\mathrm{T}_{1}$ seeds were thinner and more wrinkled than sug- $h$ seeds (Fig. 5a, e). The qRT-PCR analysis indicated that OsBEIIa transcript levels were higher in transgenic $\mathrm{T}_{1}$ plants than in sug-h mutant (Fig. 5f). These complementation data suggest that osbe2a may improve the sug-h phenotype by producing grains that are less wrinkled.

\section{Gene Expression Patterns of OsBElla}

To investigate the expression patterns of OsISA1 and OsBEIIa in different organs, we performed qRT-PCR analyses and $\beta$-glucuronidase (GUS) reporter gene assay. The qRT-PCR analyses showed that OsBEIIa was expressed primarily in leaf, stem, and seed in wild-type and sug-h mutant plants (Fig. 6a). OsBEIIa expression in 10 days after flowering (DAF) seeds was slightly downregulated in sug-h mutant compared with that in the wild type, although this change was not statistically significant. By contrast, OsISA1 expression in sug-h seeds was significantly lower than that of the wild type, and OsISA1 expression was similarly low in both wild-type and mutant leaf samples (Additional file 4: Figure S3). Transcript analysis revealed that the OsBEIIa mutation did not show typical RNA expression patterns in 


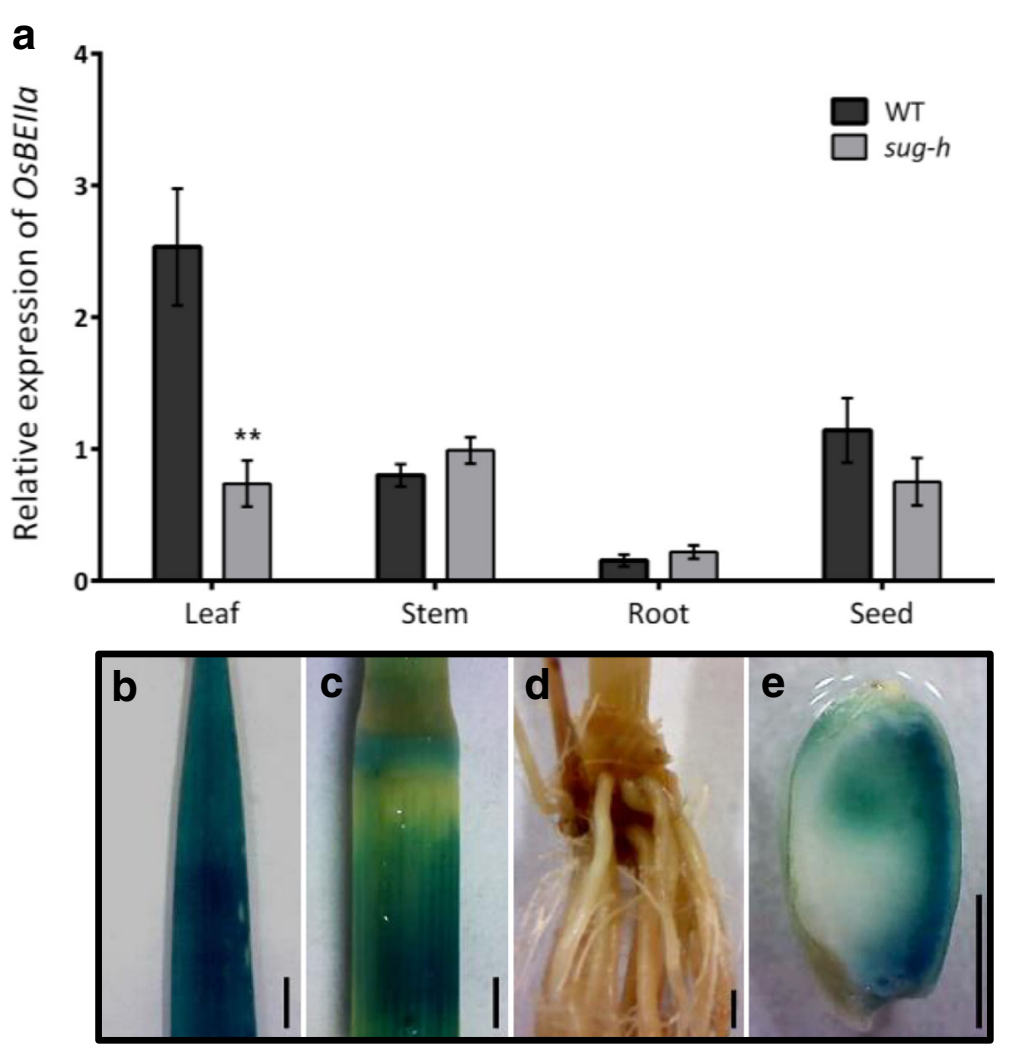

Fig. 6 Expression pattern of OsBElla gene (a) qRT-PCR analysis detected OsBElla transcripts in leaf, stem, root, and 10 DAF seeds. Data are mean \pm SD $(n=3)$. Statistical significance was determined using Student's $t$-test $\left.{ }^{* *} P<0.01\right)$. WT, wild-type rice (Hwacheong); DAF, days after flowering. b-e GUS expression was detected in leaf, root, stem base, and 20 DAF seeds in a transgenic plant expressing the OsBElla promoter::GUS reporter gene. Bars $=2 \mathrm{~mm}$

different samples, implying the possibility that other factors also determine the $s u g$ - $h$ phenotype.

GUS was expressed under the control of the native OsBEIIa promoter in the wild-type background, and the results were consistent with the qRT-PCR data. GUS expression was detected in leaf, stem base, node, and 20 DAF seeds (Fig. 6b-e). The expression levels of OsBEIIa were negligible in roots as determined by both qRT-PCR and GUS reporter analyses.

\section{Debranching and Branching Enzyme Activities in Mutant Endosperm}

Native-PAGE/DBE and BE activity staining was performed to determine the change of enzyme activity in the sug- $h$ mutant. Debranching enzymes, ISA and PUL, were detected as blue bands on the native gel containing potato tuber amylopectin stained with an iodine solution. ISA was visualized as three major bands with low mobility. ISA activity conspicuously decreased in both sugary mutants, as the same result with mapping on chromosome 8 (Fig. 7a).

Branching enzymes, BEI and BEII, were also detected on the native gel in distinct band patterns by iodine staining. BEI and BEIIb/Pho activities were shown in both wild-type and sug-1 mutant. However, BEIIa activity shown as reddish bands, dramatically decreased only in the sug-h mutant (Fig. 7b). Zymogram results imply that the difference between sug-1 and sug-h mutant was caused by branching enzyme activity.

\section{Discussion}

Genetic mapping of the sug-h rice mutant was used to identify and isolate two recessive genes, OsISA1 and OsBEIIa. The sug- $h$ mutant has a mild sugary phenotype, which preferentially accumulates desirable sugar compositions of non-starch polysaccharide rather than starch, and is more commercially viable than the sug- 1 mutant because it does not display excessive wrinkling, which interferes with milling (Koh and Heu 1994; Yoon et al. 2009). Segregation ratios of the $F_{2}$ population showed that the $s u g$ - $h$ phenotype was controlled by complementary interactions between OsISA1 and OsBEIIa. We demonstrated that OsISA1 and OsBEIIa were associated with the genetic modifications that were responsible for the sugary endosperm phenotype. Although a single mutation in OsBEIIa did not affect endosperm phenotype, 


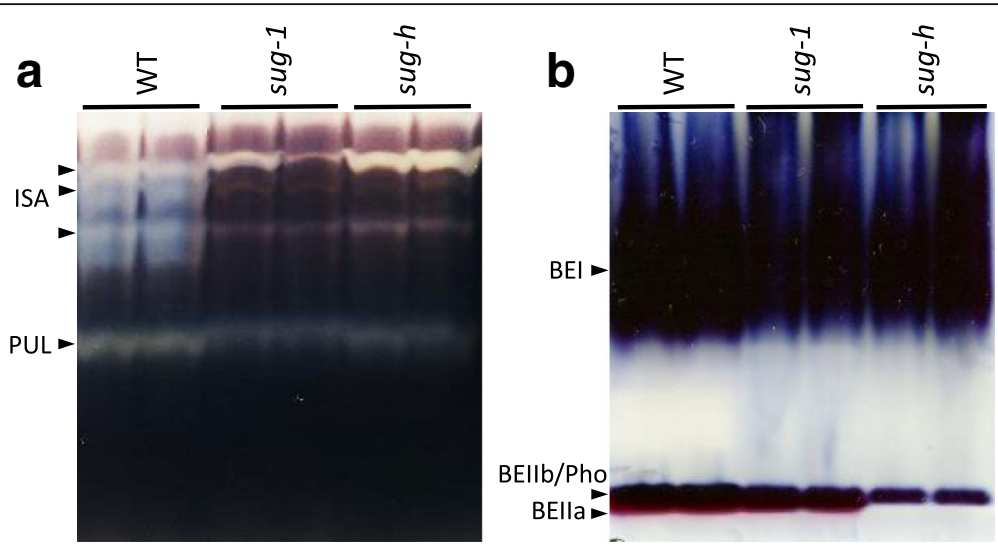

Fig. 7 Native-PAGE/activity staining of developing endosperm in wild-type and mutant seeds. a Native-PAGE/debranching enzyme (DBE) activity staining of rice endosperm at late-milky stage. The ISA and PUL activity bands are indicated by black arrows. b Native-PAGE/branching enzyme (BE) activity staining. The BEl, BElla and BEllb/Pho activity bands are indicated by black arrows

the mutation in OsBEIIa moderately recovered the sugary endosperm from the severe wrinkling caused by osisa1. Therefore, sug- $h$ mutant seed maintains a sugary phenotype, but the seed quality is superior (less wrinkled) than that of sug-1.

There have been several reported mutants and transgenic rice related to sugary-type endosperm. Among them, severe sugary-type endosperm mutants, such as EM914 (Nakamura et al. 1997; Wong et al. 2003), \#1-1 (Tanaka et al. 2004), and OsISA1 suppression and OsISA2 over-expression transgenic lines (Utsumi et al. 2011), were similar to the sug-1 mutant used in this study. Of them, phenotype of EM914 was governed by mutated ISA1 while \#1-1 and OsISA2 over-expression line had different genes than sug-1. It is interesting that a mild sugary mutant, which was reported as a variation of $s u-1$ mutant by Nakamura et al. (1997), had the similar phenotype to sug-h mutant although only sug- 1 locus was involved in the $s u-1$ mutant. The reason for the phenotypic similarity between $s u-1$ mutant by Nakamura et al. (1997) and sug-h mutant in this study remains to be comparatively studied. Recently, a rice novel endosperm mutant, named as sugary-2, was reported (Nakagami et al. 2016), in which the results on the activity of BE in the sugary-2 mutant was unlike the sug- $h$ mutant, indicating that the sugary- $2 \mathrm{mu}$ tant was different from the $s u g-h$ mutant.

Nakamura (2002) reported that inhibition of BEIIa activity caused low levels of short amylopectin chains with degree of polymerization (DP) $\leq 10$ in rice leaf sheath, in which BEIIb is not expressed; however, the BEIIa-deficient mutant does not exhibit a significant change in amylopectin chain length profile in rice endosperm. Because a direct function of the OsBEIIa could not have been elucidated yet, we suggest that the amino acid substitutions in OsISA1 and OsBEIIa changed the protein complex or enzyme interaction involved in starch biosynthesis, and might be responsible for the sug-h phenotype affecting amylopectin structure. Future studies will perform enzymatic analyses to test this hypothesis.

Previous studies investigated possible interactions between ISA and other enzymes. The debranching enzyme PUL was related to the sugary phenotype (Fujita et al. 2009). Amylose content, seed morphology, and starch granules of pul mutant lines were essentially the same as those of wild-type plants. By contrast, double pul and isa1 mutant lines contained higher levels of WSPs and had shorter amylopectin chains with DP $\leq 7$ in the endosperm compared with the sug- 1 parents, indicating that PUL can partly compensate for starch biosynthesis. The absence of ISA activity primarily affected the sugary endosperm phenotype regardless of the presence of PUL activity. This result was very similar using the sug- $h$ mutant; however, no differences in OsPUL sequences were identified in the Hwacheong wild type and the sug-h mutant.

FLOURY ENDOSPERM6 (FLO6) encodes a CBM48 domain-containing protein (Peng et al. 2014). FLO6 may act as a starch-binding protein interacting with ISA1, and may be a bridge between ISA1 and starch during starch biosynthesis. ISA1 may have interacting factors that mediate starch binding, and interacting enzymes that have not yet been elucidated. Previous research evaluated proteinprotein interactions of starch biosynthetic enzymes. Crofts et al. (2015) reviewed that co-immunoprecipitation analysis revealed the following associations in rice: BEIIa-BEIIb, BEIIa-BEI, BEIIa-Pho1, BEIIa-SSI, and BEIIa-SSIIIa. The BEIIa-SSI interaction was also identified in wheat and maize (Tetlow et al. 2008). These results suggested that some isozymes involved in starch biosynthesis in rice formed active protein complexes. These combined results suggest a possible mechanism of BEIIa function in rice endosperm.

Phenotypic variation is a critical consideration for phenotypic analysis of sugary endosperm in $s u g-h$ 
populations because of environmental effects. Satoh et al. (2008) evaluated the effect of growth temperature on the frequency of various grain phenotypes and the extent of starch accumulation in the wild type and mutant, and reported that starch accumulation in the phosphorylase1 (pho1) mutant endosperm was affected by temperature. Similarly, the seed phenotypes of sug- 1 and sug-h mutants differed slightly between plants grown in the field and those grown in the green house (data not shown). To reduce this phenotypic variation, all seeds from a whole main panicle of $F_{2}$ and $F_{3}$ plants grown in the field were used for genetic mapping. The mutants and wild-type seeds were grown together under the same conditions and prepared for phenotypic analysis at the same time. Future studies should assess the effects of environmental factors, especially temperature, on phenotypic variation.

In this study, we propose that mutated OsBEIIa plays a role in restoring the severely wrinkled sugary phenotype caused by osisa 1 in rice endosperm, although OsBEIIa mutation alone did not result in a significant phenotypic change. The observed complementary interaction between OsISA1 and OsBEIIa provides novel insight into the roles of starch biosynthesis enzymes and their interactions. Our result can facilitate the breeding of functional rice cultivars with special nutritional qualities, and might be applicable to endosperm modification in other cereal crops.

\section{Conclusions}

Grains of sug-h mutant have less wrinkled feature, which make it more commercially feasible by easy polishing in processing. Here, we report the cloning of a new sugary gene in rice, which controls the thickness of sugary endosperm grains. Genetic analysis revealed that phenotype of sug- $h$ mutant was controlled by a complementary interaction of two recessive genes, OsBEIIa and OsISA1. Complementation test demonstrated that sugary endosperm was modulated by OsBEIIa. These results may facilitate the breeding of sugary endosperm rice for commercial use and will be helpful to enlarge our understanding on starch biosynthesis in rice endosperm.

\section{Methods}

\section{Plant Materials}

The sug-h mutant was induced by N-methyl-N-nitrosourea (MNU) treatment on the Korean japonica cultivar Hwacheong (Koh and Heu 1994; The sug-2 was renamed in this study as $s u g-h$ to avoid confusion with Nakagami et al. (2016)). The $F_{2}$ population was derived from a cross between the sug-h mutant and M.23. $F_{3}$ seeds were classified into three groups: normal, sugary, and heterozygous type. Two $F_{2}$ individuals that displayed the sugary-type in $F_{3}$ seeds were developed for the $F_{3}$ population. To calculate the segregation ratio, another $F_{2}$ population derived from a cross between the sug- $h$ mutant and wild-type Hwacheong was used. The sug-1 mutant line was selected from the sug-h/Hwacheong $\mathrm{F}_{2}$ population, and isolated to the $\mathrm{F}_{6}$ generation via self-pollination. Normal-type seeds were designated as ' $\mathrm{N}$-type' and sugary-type seeds were designated as 'S-type'. S-type seeds were grouped into 'I-type' (sugary-1 type), 'II-type' (sugary-h type) and mixed-type (I-type and II-type) in segregating populations. These populations were cultivated using conventional methods at the Experimental Farm of Seoul National University.

\section{Phenotypic Analysis}

All $F_{1}, F_{2}$, and $F_{3}$ seeds were dehulled and observed under a microscope. $\mathrm{S}$-type and $\mathrm{N}$-type seeds were distinguished by seed thickness and severity of wrinkling. All seeds from a whole panicle were used for phenotyping to minimize phenotypic error resulting from differences in seed maturity. Grain dimensions including length, width, and thickness were measured using digimatic calipers (Mitutoyo, Japan) and analyzed using the IBM SPSS statistics program. Each seed was stained with iodine solution $[0.1 \%$ $(w / v) \mathrm{I}_{2}$ and $\left.1 \%(w / v) \mathrm{KI}\right]$ to detect starch.

\section{Scanning Electron Microscopy}

Seeds and starch samples were visualized with a SEM according to the previously published method of Fujita et al. (2003). Gold-coated seeds and starch samples were observed using a SUPRA 55VP Scanning Electron Microscope (Carl Zeiss, Germany).

\section{X-ray Diffraction Analysis of Starch}

One of the samples for morphological analysis of starch properties, Sug-1/sug-h, possessed normal OsISA1 and mutated osbe $2 a$ alleles. X-ray diffraction was used to determine the structures of starch according to the methods described previously by Kubo et al. (2005). The X-ray diffraction patterns of isolated insoluble glucans were obtained with a copper (nickel foil-filtered) $K_{\alpha}$ radiation using D8 Advanced Xray diffractometry (Bruker, Germany) at $40 \mathrm{kv}$ and $40 \mathrm{~mA}$. The scanning region of the two-theta angle $(2 \theta)$ ranged from 4.0 to $40.0^{\circ}$ with a scan speed of $0.5 \mathrm{deg}$. $\mathrm{min}^{-1}$.

\section{Chain-Length Distribution of Amylopectin by HPAEC-PAD} The chain-length distributions of amylopectin from wildtype and mutants endosperm were analyzed using HPAEC-PAD as in previous reports (Hanashiro et al. 1996; Kwak et al. 2017).

\section{DNA Extraction and PCR Amplification}

Total genomic DNA was extracted from young leaves of $\mathrm{F}_{2}, \mathrm{~F}_{3}$ plants and their parents according to the method of McCouch et al. (1988) with modifications. Polymerase chain reactions (PCR) were performed in a reaction volume of $20 \mu \mathrm{l}$ containing $100 \mathrm{ng}$ of template DNA, $0.1 \mu \mathrm{M}$ each primer, $2.5 \mathrm{mM} \mathrm{dNTP}, 10 \mathrm{mM}$ Tris- $\mathrm{HCl}$ 
(pH 8.3), $50 \mathrm{mM} \mathrm{KCl}, 1.5 \mathrm{mM} \mathrm{MgCl} 2,0.01 \%(w / v)$ gelatin, and $0.5 \mathrm{U}$ Taq DNA polymerase. PCR amplification was carried out in a DNA Engine Tetrad 2 and Dyad Thermal Cycler (Bio-Rad, USA) using the following reaction conditions: $5 \mathrm{~min}$ at $94{ }^{\circ} \mathrm{C}$; followed by 35 cycles of $1 \mathrm{~min}$ at $94{ }^{\circ} \mathrm{C}, 30 \mathrm{~s}$ at $56{ }^{\circ} \mathrm{C}$, and $40 \mathrm{~s}$ at $72{ }^{\circ} \mathrm{C}$; and $10 \mathrm{~min}$ at $72{ }^{\circ} \mathrm{C}$ for final extension. PCR products were separated on $2.5 \%(w / v)$ agarose gels containing $0.15 \mathrm{\mu g} \mathrm{ml}^{-1}$ ethidium bromide $(\mathrm{EtBr})$ in $0.5 \times$ TBE buffer.

\section{Linkage Mapping of the sug-h Mutant}

We performed BSA to genetically map and isolate genes related to the sug-h mutant (Michelmore et al. 1991). Ten N-type plants, ten heterozygous type plants (N-type and S-type), and ten S-type plants were selected from the sug-h/M.23 $\mathrm{F}_{2}$ population for the first BSA to identify gene distinguishing $\mathrm{N}$-type and $\mathrm{S}$-type. For the second BSA, ten I-type plants, 12 mixed-type plants (I-type and II-type), and 12 II-type plants were selected from the sug-h/M.23 $\mathrm{F}_{3}$ population, in which the sugary allele was fixed. Three bulked samples containing randomly combined equal amounts of DNA were used for genotyping. Then, fine mapping was conducted on two chromosomes with flanking STS markers, which were developed by designing primers based on the DNA sequence differences between indica and japonica rice cultivars (Chin et al. 2007). Additional STS and derived cleaved amplified polymorphic sequence (dCAPS) primers were designed with Primer3 version 0.4.0 (http://frodo.wi. mit.edu/primer3) for additional fine mapping based on the available rice genome sequence data (http://www. gramene.org, http://www.ncbi.nlm.nih.gov). Primer sequences and other information for DNA markers designed and used in this study are listed in Additional file 5: Table S2.

\section{Candidate Gene Analysis}

To validate the candidate gene models, full-length genomic DNA of each candidate gene in Hwacheong and the sug- $h$ mutant was amplified by PCR. PCR products were purified using the PCR purification kit (Inclone, Korea) and transformed into E. coli strain DH5 $\alpha$, followed by ligation of PCR amplicons into the pGEM-T Easy Vector (Promega, USA). Transformed plasmid sequences were analyzed with an ABI Prism $3730 \mathrm{XL}$ DNA Analyzer (PE Applied Biosystems, USA). The PCR clones were verified by sequence alignment with the original parent using CodonCode Aligner software (CodonCode Corporation, USA). Based on the results of the sequencing analysis, multiple alignments were performed using a public database (http://www.ch.embnet. org/software/BOX_form.html).
RNA Isolation and Quantitative Real-Time PCR Analysis Total RNA was extracted from various tissues of wild-type, mutant, and transgenic plants using MG Total RNA Extraction kit (Doctor Protein, Korea). The RNAs were converted into first-strand cDNA using M-MLV Reverse Transcriptase (Promega, USA). Quantitative RT-PCR was performed using SYBR Premix ExTaq (Takara Bio, Japan) according to the manufacturer's instructions. Gene expression levels were evaluated in leaf, stem, and root samples collected at maximum tillering stage and seed samples collected at 5, 10, and $20 \mathrm{DAF}$. The following gene-specific primer sets were used: OsISA1-RT, 5'-CAAATGCG CAATTTCTTTGTT-3' (sense) and 5'-GTCCCAGCG GAAATAATTGA-3' (antisense); OsBEIIa-RT, 5'-GCCAA TGCCAGGAAGATGA-3' (sense) and 5'-GCGCAACA TAGGATGGGTTT-3' (antisense) (Zhang et al. 2012); control UBQ-qPCR, 5'-GAGCCTCTGTTCGTCAAGTA-3' and 5' -ACTCGATGGTCCATTAAACC-3' (Tanaka et al. 2011). Quantitative RT-PCR was performed using a C1000 thermal cycler (Bio-Rad, USA).

\section{Complementation of the sug- $h$ Mutant}

The RNA interference (RNAi) vector was constructed by PCR amplification of a 291 bp fragment from OsISA1 and a 206 bp fragment from OsBEIIa cloned from Hwacheong cDNA. These fragments were subcloned into pDONR201 (Invitrogen, USA), and then transferred into the RNAi vector pH7GWIWG (II) using Gateway BP and LR clonase enzyme mixes (Invitrogen, USA). The full-length OsBEIIa cDNA was amplified from Hwacheong cDNA and used for constructing the overexpression vector. The amplified fragment was transferred into pMDC32 via $\mathrm{pCR}^{\mathrm{m}}$ 8/GW/TOPO ${ }^{\circ}$ TA Cloning Kit (Invitrogen, USA). The RNAi constructs were transformed into wild-type Dongjin (a japonica cultivar), and the overexpression construct was transformed into callus of the sug-h mutant. Transformation was performed using a modification of the previously published Agrobacterium-mediated transformation method (Nishimura et al. 2006).

\section{Histological GUS Assay}

The 1909 bp region upstream from the start codon of OsBEIIa was amplified and cloned into the binary vector pHGWFS7 using Gateway BP and LR clonase enzyme mixes (Invitrogen, USA). The final construct was introduced into wild-type Dongjin by Agrobacterium-mediated transformation. Transgenic plants containing the OsBEIIa promoter::GUS reporter construct were selected, and $\mathrm{T}_{0}$ plants were used for GUS assays. GUS staining was performed as described previously (Jefferson et al. 1987). $\mathrm{X}$-Gluc buffer solution was vacuum-infiltrated into several different tissue samples. The samples were incubated overnight in X-Gluc buffer solution at $37{ }^{\circ} \mathrm{C}$, and then washed with a graded ethanol series. 
Native-PAGE/Activity Staining of DBE and BE Crude enzyme was extracted using the method described by Yamanouchi and Nakamura (1992) from ten seeds at late-milky stage. Native-PAGE/activity staining of DBE was performed using the modified method of Nakamura et al. (1997). Native slab gel was prepared with $6.5 \%(w / v$; resolving gel) containing $0.3 \%(w / v)$ potato tuber amylopectin (Sigma, USA), and $3.3 \%(w / v$; stacking gel) acrylamide. Electrophoresis was carried out at $4{ }^{\circ} \mathrm{C}$ at constant current of $20 \mathrm{~mA}$ for $2.5 \mathrm{~h}$. For detection of the isoamylase activity, the gel was rinsed with $20 \mathrm{ml}$ of $50 \mathrm{mM} \mathrm{Na}$-acetate (pH 5.4), $50 \mathrm{mM}$ 2-mercaptoethanol and $50 \mathrm{mM} \mathrm{CaCl}_{2}$ at room temperature and then incubated at $37{ }^{\circ} \mathrm{C}$ for $3 \mathrm{~h}$ with $20 \mathrm{ml}$ of the same buffer solution. NativePAGE/activity staining of BE was carried out according to the modified method by Yamanouchi and Nakamura (1992). A slab gel prepared with 5\% (w/v; resolving gel) and 3.3\% (w/v; stacking gel) acrylamide. Electrophoresis was carried out at $4{ }^{\circ} \mathrm{C}$ at constant current of $20 \mathrm{~mA}$. After electrophoresis, the gel was imbibed with $20 \mathrm{~mL}$ of a solution containing $50 \mathrm{mM}$ HEPES-NaOH buffer $(\mathrm{pH} 7.4)$ and $20 \%(\mathrm{v} / \mathrm{v})$ glycerol for $15 \mathrm{~min}$ on ice. For detection of BE, the gel was incubated in $20 \mathrm{ml}$ of the reaction mixture, which consisted of $50 \mathrm{mM}$ HEPES$\mathrm{NaOH}$ buffer (pH 7.4), $50 \mathrm{mM}$ Glc-1-P (Sigma, USA), $2.5 \mathrm{mM}$ AMP (Sigma, USA), 10\% $(v / \mathrm{v})$ glycerol, and rabbit muscle phosphorylase a (about 30 units; Sigma, USA) for $6 \mathrm{~h}$ at $30{ }^{\circ} \mathrm{C}$ with gentle shaking. Iodine solution $\left[0.1 \%(w / v) \mathrm{I}_{2}\right.$ and $\left.1 \%(w / v) \mathrm{KI}\right]$ was used for staining both gels.

\section{Additional files}

Additional file 1: Figure S1. Plant and panicle morphology of wild-type rice (Hwacheong) and mutants. (a-b) Plant phenotype of wild-type and mutant plants 54 days after transplanting (a) and at the milky stage (b). (c) Panicle length of wild-type and mutant plants at the yellow ripe stage. (PDF $439 \mathrm{~kb}$ )

Additional file 2: Table S1. Genetic analysis of the sug-h mutant using $F_{3}$ seeds (DOCX $14 \mathrm{~kb}$ )

Additional file 3: Figure S2. Multiple alignments of cereal OsBElla proteins. Protein of the sug-h mutant (top line) was aligned with that of wild-type rice (Hwacheong) and four cereal plants (barley, maize, sorghum, and wheat). Black boxes indicate identical residues; gray boxes indicate similar residues. Mutated region is marked with an asterisk. Color bars indicate the domains; E_set_GBE_euk_N (green), AmyAc_baC_euk_BE (blue), and Alpha-amylase_C (orange). (PDF 1064 kb)

Additional file 4: Figure S3. OsISA1 expression patterns in different organs and at different stages of seed development using GRT-PCR analysis. (a) Transcript levels decreased in seed (10 DAF) of the sug-h mutant. (b) OsISA1 expression in 10 and 20 DAF seeds decreased in the sug-h mutant. All data are mean $\pm \mathrm{SD}(n=3)$. Statistical significance was determined using Student's $t$-test $\left.{ }^{*} P<0.05,{ }^{*} P<0.01\right)$. WT, wild-type rice (Hwacheong); DAF, days after flowering. (PDF $51 \mathrm{~kb}$ )

Additional file 5: Table S2. Molecular markers used for fine mapping of the sug-h mutant. (DOCX $14 \mathrm{~kb}$ )

\section{Abbreviations}

BElla: Branching enzyme Ila; BSA: Bulked segregant analysis; dCAPS: Derived cleaved amplified polymorphic sequence; ISA1: Isoamylase1; PCR: Polymerase chain reaction; STS: Sequence tagged site; sug-1: sugary-1; sug-h: sugary-h; WSP: Water-soluble polysaccharide

\section{Acknowledgments}

Authors express sincere thanks to Prof. Fujita in Akita Prefectural University, Japan, for her kind advice on native-PAGE/BE staining. Authors also thank Dr. Joohyun Lee in Konkuk University and Dr. Soon-Wook Kwon in Pusan National University for supporting material development and helpful discussion. This study was supported by a grant from the Next-Generation BioGreen 21 Program (no. PJ01102401) of the Rural Development Administration, Korea.

\section{Authors' Contributions}

HJK and JHC supervised the work and improved the manuscript. YL designed and performed experiments, and wrote the manuscript. MSC developed the plant materials, and performed experiments. GL, SJ, BK, RP provided advice on the experiments. MRY supported experimental data. MOW improved the manuscript. All authors reviewed the manuscript. All authors read and approved the final manuscript.

\section{Competing Interests}

The authors declare that they have no potential competing interests.

\section{Publisher's Note}

Springer Nature remains neutral with regard to jurisdictional claims in published maps and institutional affiliations.

\section{Author details}

'Department of Plant Science and Research Institute for Agriculture and Life Sciences, and Plant Genomics and Breeding Institute, Seoul National University, Seoul 08826, South Korea. ${ }^{2}$ Vegetable Crop Division, National Institute of Horticultural and Herbal Science, Rural Development Administration, Muan 534-833, South Korea. ${ }^{3}$ Department of Central Area Crop Science, National Institute of Crop Science (NICS), RDA, Suwon 16429, South Korea. ${ }^{4}$ Rice Research Institute, Jilin Academy of Agricultural Sciences, Gongzhuling, Jilin 136100, China. ${ }^{5}$ Graduate School of Integrated Bioindustry, Sejong University, 209, Neungdong-ro Gwangjin-gu, Seoul, South Korea.

Received: 21 February 2017 Accepted: 10 July 2017 Published online: 20 July 2017

\section{References}

Chin J-H, Kim J-H, Jiang W, Chu S-H, Woo M-O, Han L, Brar D, Koh H-J (2007) Identification of subspecies-specific STS markers and their association with segregation distortion in rice (Oryza sativa L.) J Crop Sci Biotec 10:175-184

Crofts N, Abe N, Oitome NF, Matsushima R, Hayashi M, Tetlow IJ, Emes MJ, Nakamura Y, Fujita N (2015) Amylopectin biosynthetic enzymes from developing rice seed form enzymatically active protein complexes. J Exp Bot 66:4469-4482

Fujita N (2014) Starch biosynthesis in Rice endosperm. AGBM 4:1-18

Fujita N, Kubo A, Francisco PB Jr, Nakakita M, Harada K, Minaka N, Nakamura Y (1999) Purification, characterization, and CDNA structure of isoamylase from developing endosperm of rice. Planta 208:283-293

Fujita N, Kubo A, Suh DS, Wong KS, Jane JL, Ozawa K, Takaiwa F, Inaba Y, Nakamura Y (2003) Antisense inhibition of isoamylase alters the structure of amylopectin and the physicochemical properties of starch in rice endosperm. Plant Cell Physiol 44:607-618

Fujita N, Toyosawa Y, Utsumi Y, Higuchi T, Hanashiro I, Ikegami A, Akuzawa S, Yoshida M, Mori A, Inomata K, Itoh R, Miyao A, Hirochika H, Satoh H, Nakamura Y (2009) Characterization of pullulanase (PUL)-deficient mutants of rice (Oryza sativa L.) and the function of PUL on starch biosynthesis in the developing rice endosperm. J Exp Bot 60:1009-1023

Hanashiro I, J-i A, Hizukuri S (1996) A periodic distribution of the chain length of amylopectin as revealed by high-performance anion-exchange chromatography. Carbohydr Res 283:151-159

James MG, Denyer K, Myers AM (2003) Starch synthesis in the cereal endosperm. Curr Opin Plant Biol 6:215-222 
Jefferson RA, Kavanagh TA, Bevan MW (1987) GUS fusions: beta-glucuronidase as a sensitive and versatile gene fusion marker in higher plants. EMBO J 6:3901-3907

Kim K-N, Fisher DK, Gao M, Guiltinan MJ (1998) Molecular cloning and characterization of the Amylose-extender gene encoding starch branching enzyme IIB in maize. Plant Mol Biol 38:945-956

Koh HJ, Heu MH (1994) Physicochemical properties of sugary-endosperm mutants in Rice. Korean J Crop Sci 39:1-6

Kubo A, Fujita N, Harada K, Matsuda T, Satoh H, Nakamura Y (1999) The starch-debranching enzymes isoamylase and pullulanase are both involved in amylopectin biosynthesis in rice endosperm. Plant Physiol 121:399-410

Kubo A, Rahman S, Utsumi Y, Li Z, Mukai Y, Yamamoto M, Ugaki M, Harada K, Satoh H, Konik-Rose C, Morell M, Nakamura Y (2005) Complementation of sugary-1 phenotype in rice endosperm with the wheat isoamylase1 gene supports a direct role for isoamylase1 in amylopectin biosynthesis. Plant Physiol 137:43-56

Kwak J, Yoon M-R, Lee J-S, Lee J-H, Ko S, Tai TH, Won Y-J (2017) Morphological and starch characteristics of the japonica rice mutant variety Seolgaeng for dry-milled flour. Food Sci and Biotech 26:43-48

Lin Q, Huang B, Zhang M, Zhang X, Rivenbark J, Lappe RL, James MG, Myers AM, Hennen-Bierwagen TA (2012) Functional interactions between starch synthase III and isoamylase-type starch-debranching enzyme in maize endosperm. Plant Physiol 158:679-692

Mccouch SR, Kochert G, Yu ZH, Wang ZY, Khush GS, Coffman WR, Tanksley SD (1988) Molecular mapping of Rice chromosomes. Theor Appl Genet $76: 815-829$

Michelmore RW, Paran I, Kesseli RV (1991) Identification of markers linked to disease-resistance genes by bulked segregant analysis: a rapid method to detect markers in specific genomic regions by using segregating populations. Proc Natl Acad Sci U S A 88:9828-9832

Mizuno K, Kobayashi E, Tachibana M, Kawasaki T, Fujimura T, Funane K, Kobayashi M, Baba T (2001) Characterization of an Isoform of Rice starch branching enzyme, RBE4, in developing seeds. Plant Cell Physiol 42:349-357

Nakagami T, Yoshihara H, Nakamura T, Utsumi Y, Sawada T, Fujita N, Satoh H, Nakamura $Y$ (2016) Biochemical analysis of new type mutants of japonica rice that accumulate water-soluble a-glucans in the endosperm but retain full starch debranching enzyme activities. Starch-Stärke 68:1-11

Nakamura Y (2002) Towards a better understanding of the metabolic system for amylopectin biosynthesis in plants: rice endosperm as a model tissue. Plant Cell Physiol 43:718-725

Nakamura Y, Kubo A, Shimamune T, Matsuda T, Harada K, Satoh H (1997) Correlation between activities of starch debranching enzyme and alpha-polyglucan structure in endosperms of sugary-1 mutants of rice. Plant J 12:143-153

Nakamura Y, Umemoto T, Takahata Y, Amano E (1992) Characteristics and roles of key enzymes associated with starch biosynthesis in rice endosperm. Gamma Field Symposia 31:25-44

Nakamura Y, Umemoto T, Takahata Y, Komae K, Amano E, Satoh H (1996) Changes in structure of starch and enzyme activities affected by sugary mutations in developing rice endosperm. Possible role of starch debranching enzyme (R-enzyme) in amylopectin biosynthesis. Physiol plantarum 97:491-498

Nishi A, Nakamura Y, Tanaka N, Satoh H (2001) Biochemical and genetic analysis of the effects of Amylose-extender mutation in Rice endosperm. Plant Physiol 127:459-472

Nishimura A, Aichi I, Matsuoka M (2006) A protocol for Agrobacterium-mediated transformation in rice. Nat Protoc 1:2796-2802

Ohdan T, Francisco PB, Sawada T, Hirose T, Terao T, Satoh H, Nakamura Y (2005) Expression profiling of genes involved in starch synthesis in sink and source organs of rice. J Exp Bot 56:3229-3244

Peng C, Wang Y, Liu F, Ren Y, Zhou K, LV J, Zheng M, Zhao S, Zhang L, Wang C, Jiang L, Zhang X, Guo X, Bao Y, Wan J (2014) FLOURY ENDOSPERM6 encodes a CBM48 domain-containing protein involved in compound granule formation and starch synthesis in rice ENDOSPERM. Plant J 77:917-930

Regina A, Bird A, Topping D, Bowden S, Freeman J, Barsby T, Kosar-Hashemi B, Li Z, Rahman S, Morell M (2006) High-amylose wheat generated by RNA interference improves indices of large-bowel health in rats. Proc Natl Acad Sci U S A 103:3546-3551

Satoh H, Shibahara K, Tokunaga T, Nishi A, Tasaki M, Hwang SK, Okita TW, Kaneko N, Fujita N, Yoshida M, Hosaka Y, Sato A, Utsumi Y, Ohdan T, Nakamura Y (2008) Mutation of the plastidial alpha-glucan phosphorylase gene in rice affects the synthesis and structure of starch in the endosperm. Plant Cell 20:1833-1849
Tanaka N, Fujita N, Nishi A, Satoh H, Hosaka Y, Ugaki M, Kawasaki S, Nakamura Y (2004) The structure of starch can be manipulated by changing the expression levels of starch branching enzyme $\mathrm{llb}$ in rice endosperm. Plant Biotechnol J 2:507-516

Tanaka N, Itoh H, Sentoku N, Kojima M, Sakakibara H, Izawa T, Itoh J, Nagato Y (2011) The COP1 ortholog PPS regulates the juvenile-adult and vegetative-reproductive phase changes in rice. Plant Cell 23:2143-2154

Tetlow IJ, Beisel KG, Cameron S, Makhmoudova A, Liu F, Bresolin NS, Wait R, Morell MK, Emes MJ (2008) Analysis of protein complexes in wheat amyloplasts reveals functional interactions among starch biosynthetic enzymes. Plant Physiol 146:1878-1891

Utsumi Y, Utsumi C, Sawada T, Fujita N, Nakamura Y (2011) Functional diversity of isoamylase oligomers: the ISA1 homo-oligomer is essential for amylopectin biosynthesis in rice endosperm. Plant Physiol 156:61-77

Wong K-S, Kubo A, J-I J, Harada K, Satoh H, Nakamura Y (2003) Structures and properties of amylopectin and phytoglycogen in the endosperm of sugary-1 mutants of rice. J Cereal Sci 37:139-149

Yamanouchi H, Nakamura Y (1992) Organ specificity of isoforms of starch branching enzyme (Q-enzyme) in rice. Plant Cell Physiol 33:985-991

Yano M, Isono Y, Satoh H, Omura T (1984) Gene analysis of sugary and shrunken mutants of rice, Oryza sativa L. Jpn J Breed 34:43-49

Yoon M-R, Koh H-J, Lee S-C, Kang M-Y (2009) Comparative study of the physicochemical properties of Rice endosperm components expressing sugary-2 mutant in different genetic backgrounds. J Korean Soc Appl Biol Chem 52:509-515

Zhang M-Z, Fang J-H, Yan X, Liu J, Bao J-S, Fransson G, Andersson R, Jansson C Åman P, Sun C (2012) Molecular insights into how a deficiency of amylose affects carbon allocation-carbohydrate and oil analyses and gene expression profiling in the seeds of a rice waxy mutant. BMC Plant Biol 12:1-18

\section{Submit your manuscript to a SpringerOpen ${ }^{\circ}$ journal and benefit from:}

- Convenient online submission

- Rigorous peer review

- Open access: articles freely available online

- High visibility within the field

Retaining the copyright to your article

Submit your next manuscript at springeropen.com 